УДК $517.55+517.9$

\title{
On Formal Solutions of Hörmander's Initial-boundary Value Problem in the Class of Laurent Series
}

\author{
Evgeny K. Leinartas* \\ Tatiana I. Yakovleva ${ }^{\dagger}$ \\ Institute of Mathematics and Computer Science \\ Siberian Federal University \\ Svobodny, 79, Krasnoyarsk, 660041
}

Russia

Received 22.12.2017, received in revised form 25.12.2017, accepted 20.02.2018

We define a derivation of the ring of Laurent series with supports in rational cones and prove existence and uniqueness of a solution to an analog of one initial-boundary value problem of Hörmander for polynomial differential operators with constant coefficients in the class of formal Laurent series.

Keywords: differential operator, Hörmander's problem, difference equations, multiple Laurent series. DOI: 10.17516/1997-1397-2018-11-3-278-285.

\section{Introduction and preliminaries}

A large number of papers have been devoted to various versions of generalization of the Cauchy-Kovalevskaya theorem. Note the paper [1], which deals with the equation solved for mixed derivative, and also papers [2,3], in which systems of linear partial differential equations and existence and uniqueness theorems for normal and analytic solutions of these systems were considered. In the paper of L. Hörmander [4], in connection with the investigation of the Cauchy problem, the following initial-boundary value problem is formulated.

Let us consider a polynomial differential operator of the form

$$
P(\partial, \xi)=\partial^{m}-\sum_{\|\omega\| \leqslant d} c_{\omega}(\xi) \partial^{\omega}
$$

where $\omega=\left(\omega_{1}, \ldots, \omega_{n}\right), m=\left(m_{1} \ldots, m_{n}\right)$ are multi-indices, $\|\omega\|=\omega_{1}+\cdots+\omega_{n},\|m\|=d$, $\partial=\left(\partial_{1}, \ldots, \partial_{n}\right), \partial_{j}$ are partial derivatives, the coefficients $c_{\omega}(\xi)$ are analytic functions of $\xi=$ $\left(\xi_{1}, \ldots, \xi_{n}\right)$ in a neighborhood of zero in the space $\mathbb{C}^{n}$. It is required to find a function satisfying the differential equation

$$
P(\partial, \xi) F=G
$$

and conditions:

$$
\left.\partial_{j}^{k}[F-\Phi]\right|_{\xi_{j}=0}=0, \quad 0 \leqslant k<m_{j}, \quad j=1, \ldots, n,
$$

where $\Phi$ and $G$ are given analytic functions in a neighborhood of zero. In [4], Theorem 5.1.1, under certain restrictions on the coefficients $c_{\omega}(\xi)$ a theorem of existence and uniqueness of an

\footnotetext{
*lein@mail.ru

$\dagger$ t.neckrasova@gmail.com

(c) Siberian Federal University. All rights reserved
} 
analytic solution of the initial-boundary value problem is obtained. The Cauchy-Kovalevskaya theorem and the Darboux-Goursat-Baudot theorem follow from this theorem.

In this paper we formulate an analogue of the problem (1)-(2), in which instead of the partial derivatives $\partial_{j}$ the operators $D_{j}$ of derivation of the ring of Laurent series with supports in rational cones are used. In the case of constant coefficients the question of existence and uniqueness of a formal solution in the class of such series is investigated.

In the second section we give notation and definitions necessary for formulation of an analog of the Hörmander problem (1)-(2), and for formulation of the theorem of existence and uniqueness of a solution to this problem (Theorem 1).

In the third section we define the Borel transform of Laurent series with supports in rational cones which allows us to establish the connection (Theorem 2) between solvability of the corresponding initial boundary value problems for differential and difference equations with identical symbols. Using the results on solvability of the difference problem obtained in [5], we prove the main Theorem 1.

\section{An analog of the initial-boundary value problem of Hörmander}

To formulate an analog of the initial-boundary value problem of Hörmander, we need the definitions of a rational cone, of the ring of Laurent series with supports in these cones and derivations of the ring of such series (see [6,7]).

Let $a^{1}, \ldots, a^{n}$ be linearly independent vectors with integer coordinates $a^{j}=\left(a_{1}^{j}, \ldots, a_{n}^{j}\right)$. $A$ rational cone generated by $a^{1}, \ldots, a^{n}$ is the set

$$
K=\left\{x \in \mathbb{R}^{n}: x=\lambda_{1} a^{1}+\cdots+\lambda_{n} a^{n}, \lambda_{j} \in \mathbb{R}_{+}, j=1, \ldots, n\right\} .
$$

We denote by $A$ the matrix whose columns consist of the coordinates of the vectors $a^{j}$ and $\Delta=\operatorname{det} A$. If $\Delta=1$, then the cone $K$ is unimodular. We shall consider only unimodular cones.

Let $A^{-1}$ be the inverse of the matrix $A$. The rows of $A^{-1}$ are denoted by $\alpha^{1}, \ldots, \alpha^{n}$. Let the vectors $\alpha^{1}, \ldots, \alpha^{n}$ form a reciprocal basis for the vectors $a^{1}, \ldots, a^{n}$, that is $\left\langle\alpha^{i}, a^{j}\right\rangle=\delta_{i j}$, where $\langle k, x\rangle=k_{1} x_{1}+\cdots+k_{n} x_{n}$, and $\delta_{i j}$ is the Kronecker symbol. Note that for $x \in K$ always $\left\langle\alpha^{j}, x\right\rangle \geqslant 0, j=1, \ldots, n$.

Define a partial order $\underset{K}{\geqslant}$ on points $u, v \in \mathbb{R}^{n}$ as follows:

$$
u \underset{K}{\geqslant} v \Leftrightarrow u-v \in K
$$

Moreover, we write $u \not 夫 v$ whenever $u-v \notin K$.

The cone

$$
K^{*}=\left\{k \in \mathbb{R}^{n}:\langle k, x\rangle \geqslant 0, x \in K\right\}
$$

is called dual to $K$. Denote the set of its interior points by $\stackrel{\circ}{K}^{*}$ and fix $\nu \in \stackrel{\circ}{K}^{*} \cap \mathbb{Z}^{n}$. Given $x \in K \cap \mathbb{Z}^{n}$, the nonnegative number

$$
|x|_{\nu}=\langle\nu, x\rangle,
$$

is referred to as the weighted homogeneous degree of $z^{x}$. The weighted homogeneous degree of the Laurent polynomial $Q(z)=\sum_{x} q_{x} z^{x}$ is defined by the formula

$$
\operatorname{deg}_{\nu} Q(z)=\max _{x}|x|_{\nu}
$$


Denote by $\mathbb{C}_{K}[z]$ the ring of Laurent polynomials $Q(z)=\sum_{x} q_{x} z^{x}$ with the exponents $x$ of the monomials $z^{x}$ lying in $K \cap \mathbb{Z}^{n}$. Addition and multiplication are defined naturally.

In the ring $\mathbb{C}_{K}[[z]]$ of Laurent series the usual partial derivative $\frac{\partial}{\partial z_{j}}$ is not a derivation because for $x \in K \cap \mathbb{Z}^{n}$ the points $x-e^{j}$, where $e^{j}$ are the standard unit vectors, in general need not lie in $K \cap \mathbb{Z}^{n}$. In [7], the derivation of the ring $\mathbb{C}_{K}[[z]]$ was defined, which made it possible to transfer the concept of D-finiteness of power series to Laurent series. We repeat this definition.

Express each element $x \in K \cap \mathbb{Z}^{n}$ as a linear combination $x=\lambda_{1} a^{1}+\cdots+\lambda_{n} a^{n}$ of basis vectors. In the matrix form this expression becomes $x=A \lambda$, where $\lambda$ is a column vector, and $A$ is the matrix with the coordinates of the vectors $a^{j}$ in columns

$$
A=\left(\begin{array}{ccc}
a_{1}^{1} & \ldots & a_{1}^{n} \\
. . & . . & . . \\
a_{n}^{1} & \ldots & a_{n}^{n}
\end{array}\right) .
$$

The determinant of matrix $A$ is not equal to zero.

Define the operator $D_{j}$ on monomials $z^{x}$ as follows:

$$
D_{j} z^{x}=\lambda_{j} z^{x-a^{j}}
$$

where $\lambda_{j}$ is the $\mathrm{j}$-th coordinate of the point $\lambda=A^{-1} x$. Observe that for $\Delta \neq 1$ and $x \in K \cap \mathbb{Z}^{n}$ the number $\lambda_{j}$ is rational in general.

It is not difficult to verify that $D_{j}$ for $j=1, \ldots, n$, are linear and satisfy the usual rule for the derivative of the product and, in the case of a unimodular cone $(\Delta=1)$, map the ring $\mathbb{C}_{K}[[z]]$ into itself, that is, they are derivations.

Next, on the monomials $z^{x}, x \in K \cap \mathbb{Z}^{n}$, define the operator $D^{\omega}, \omega \in K \cap \mathbb{Z}^{n}, \omega=\omega_{1} a^{1}+$ $+\cdots+\omega_{n} a^{n}$ as follows:

$$
D^{\omega} z^{x}=\Pi_{j}\left\langle x, \alpha^{j}\right\rangle\left\langle x-a^{j}, \alpha^{j}\right\rangle \ldots\left\langle x-\left(\omega_{j}-1\right) a^{j}, \alpha^{j}\right\rangle z^{x-\omega} .
$$

Note that $D^{\omega^{\prime}} D^{\omega^{\prime \prime}}=D^{\omega^{\prime}+\omega^{\prime \prime}}$. For $\omega=a^{j}$ we have $D^{a^{j}} z^{x}=\left\langle x, \alpha^{j}\right\rangle z^{x-a^{j}}=D_{j} z^{x}, j=1, \ldots, n$, and if $\omega_{1}, \ldots, \omega_{n}$ are the coordinates of the vector $\omega$ in the basis $a^{1}, \ldots, a^{n}$, then $D^{\omega}=D^{a^{1} \omega_{1}} \ldots$ $\ldots D^{a^{n} \omega_{n}}=D_{1}^{\omega_{1}} \ldots D_{n}^{\omega_{n}}$, where $D_{j}^{k}=\underbrace{D_{j} \circ \cdots \circ D_{j}}_{k \text { times }}$.

Note that if the cone $K$ is unimodular, then the operators $D^{\omega}$ for $\omega \in K \cap \mathbb{Z}^{n}$ are derivations of the ring of series $\mathbb{C}_{K}[[z]]$ and the action of the operator $D^{\omega}$ on the monomials $z^{x}, x \in K \cap \mathbb{Z}^{n}$, can be written as follows:

$$
D^{\omega} z^{x}=\left\{\begin{array}{cc}
0, & \text { if } x \underset{K}{\neq} \omega, x \neq \omega, \\
\frac{\langle x, \alpha\rangle !}{\langle x-\omega, \alpha\rangle !} z^{x-\omega}, & \text { if } x \geqslant \omega
\end{array}\right.
$$

where $\langle x, \alpha\rangle !=\left\langle x, \alpha^{1}\right\rangle ! \ldots\left\langle x, \alpha^{n}\right\rangle !$.

We denote by $\Gamma_{j}$ the face of the cone $K$ generated by the vectors $a^{i}, i=1, \ldots, j-1, j+1, \ldots, n$, $\Gamma_{j}=\left\{x: x=\lambda_{1} a^{1}+\ldots \lambda_{j-1} a^{j-1}+\lambda_{j+1} a^{j+1}+\cdots+\lambda_{n} a^{n}\right\}$. Denote by $\left.\mathcal{F}(z)\right|_{z^{a j}=0}$ the Laurent series, whose supports are the faces $\Gamma_{j}$ of the rational cone $K$ :

$$
\left.\mathcal{F}(z)\right|_{z^{a j}=0}=\sum_{x \in \Gamma_{j} \cap \mathbb{Z}^{n}} f(x) z^{x}
$$


Let us define a polynomial differential operator

$$
P(D)=\sum_{\omega \in \Omega} c_{\omega}(z) D^{\omega},
$$

where $\Omega \subset K \cap \mathbb{Z}^{n}$ is a finite set of points of an $n$-dimensional integer lattice and coefficients $c_{\omega} \in \mathbb{C}_{K}[[z]]$. The Laurent polynomial $P(\zeta)=\sum_{\omega \in \Omega} c_{\omega} \zeta^{\omega}$ is called the characteristic polynomial for the polynomial differential operator (6).

By the order $d_{\nu}$ of $P(D)$, we mean the weighted homogeneous degree $\operatorname{deg}_{\nu} P(\zeta)$ of the characteristic polynomial, i.e., $d_{\nu}=\max _{\omega \in \Omega}|\omega|_{\nu}$. In what follows we omit the subscript $\nu$ for $d$.

Remark. It follows from the relation

$$
z^{a^{j}} D_{j}=\left\langle\alpha^{j}, \Theta\right\rangle, j=1, \ldots, n,
$$

where $\Theta=\left(z_{1} \frac{\partial}{\partial z_{1}}, \ldots, z_{n} \frac{\partial}{\partial z_{n}}\right), \alpha^{j}, j=1, \ldots, n$, is a reciprocal basis, that the differential operator (6) can also be regarded as a differential operator with partial derivatives. As an example, consider the following operator:

$$
P(D)=c_{2,1} D^{(2,1)}+c_{1,0} D^{(1,0)}+c_{1,1} D^{(1,1)}+c_{0,0} .
$$

Taking into account (7), it can be written as

$$
c_{2,1} z^{-1} \frac{\partial^{2}}{\partial z_{1} \partial z_{2}}-c_{2,1} z_{1}^{-2} z_{2} \frac{\partial^{2}}{\partial z_{2}^{2}}+c_{1,0} \frac{\partial}{\partial z_{1}}+\left(-c_{2,1} z_{1}^{2}-c_{1,0} z_{1}^{-1} z_{2}+c_{1,1} z_{1}^{-1}\right) \frac{\partial}{\partial z_{2}}+c_{0,0} .
$$

Let us fix $m \in K \cap \mathbb{Z}^{n}$ and formulate the following problem which we call an analog of the initial-boundary value problem of Hörmander. It is required to find $\mathcal{F}(z)$, satisfying the equation

$$
P(D) \mathcal{F}=G,
$$

and initial-boundary conditions

$$
\left.D^{a^{j} k}[\mathcal{F}-\Phi]\right|_{z^{a j}=0}=0, \quad 0 \leqslant k<\left\langle m, \alpha^{j}\right\rangle, \quad j=1, \ldots, n .
$$

For $K=\mathbb{R}_{+}^{n}, D_{j}=\partial_{j}, j=1, \ldots, n$, the problem (8)-(9) was formulated in [4] and under certain restrictions on the coefficients of the homogeneous component of the highest power, the existence and uniqueness theorem was proved.

In the following theorem, in the case of constant coefficients of the polynomial differential operator $P(D)$, a sufficient condition is imposed on the coefficients of the principal symbol $P_{d}(D)=\sum_{|\omega|_{\nu}=d} c_{\omega} D^{\omega}$ of the differential operator $P(D)$, which ensures the existence and uniqueness of a solution.

Theorem 1. Let $m \in \Omega$ and let $|m|_{\nu}=d$ be the order of a differential operator. If the coefficients of operator (6) are constant and the coefficients of the principal symbol of $P_{d}(D)$ satisfy the condition

$$
\left|c_{m}\right|>\sum_{|\omega|_{\nu}=d, \omega \neq m}\left|c_{\omega}\right|,
$$

then for any series $G, \Phi \in \mathbb{C}_{K}[[z]]$, the boundary value problem (8)-(9) has a unique solution $\mathcal{F} \in \mathbb{C}_{K}[[z]]$. 


\section{Proof of the main theorem}

In this section we establish a connection between the solutions $\mathcal{F}$ of a differential equation from the ring $\mathbb{C}_{K}[[z]]$ and solutions of the difference equation.

In the one-dimensional case, the differential operator is written in the form $P(D)=\sum_{\omega=0}^{m} c_{\omega} D^{\omega}$, $D=\frac{d}{d z}$, and the problem (8)-(9) takes the form

$$
\begin{gathered}
P(D) \mathcal{F}(z)=G(z) \\
\left.D^{\omega}[\mathcal{F}-\Phi]\right|_{z=0}=0, \quad 0 \leqslant \omega<m,
\end{gathered}
$$

or, which is the same,

$$
\mathcal{F}^{(\omega)}(0)=\varphi(\omega), \omega=0,1, \ldots, m-1
$$

where $\varphi(\omega)$ are the coefficients of expansion in a series of functions $\Phi(z)=\sum_{x=0}^{\infty} \frac{\varphi(x)}{x !} z^{x}$. If $G(z)=\sum_{x=0}^{\infty} \frac{g(x)}{x !} z^{x}$, then it is not difficult to see that the function $\mathcal{F}(z)=\sum_{x=0}^{\infty} \frac{f(x)}{x !} z^{x}$ is a solution of (11)-(12) if and only if the coefficients $f(x)$ in its expansion in a power series satisfy the difference equation

$$
\sum_{\omega=0}^{m} c_{\omega} f(x+\omega)=g(x), x=0,1, \ldots
$$

with initial data

$$
f(x)=\varphi(x), x=0,1, \ldots, m-1
$$

where $\varphi(x)$ is a given function.

Note that the generating function $F=\sum_{x=0}^{\infty} \frac{f(x)}{z^{x}}$ of the solution of the difference equation (13) is the Borel transform of the solution $\mathcal{F}(z)=\sum_{x=0}^{\infty} \frac{f(x)}{x !} z^{x}$ of the differential equation (11).

We formulate a multidimensional analog of the difference problem (13)-(14). On complexvalued functions $f(x)=f\left(x_{1}, \ldots, x_{n}\right)$ of integer variables $x_{1}, \ldots, x_{n}$, we define shift operators $\delta_{j}$ with respect to variables $x_{j}$ :

$$
\delta_{j} f(x)=f\left(x_{1}, \ldots, x_{j-1}, x_{j}+1, x_{j+1}, \ldots, x_{n}\right)
$$

and a polynomial difference operator of the form

$$
P(\delta)=\sum_{\omega \in \Omega} c_{\omega} \delta^{\omega}
$$

where $\Omega \subset K \cap \mathbb{Z}^{n}$ is a finite set of points of an $n$-dimensional integer lattice, $\delta^{\omega}=\delta_{1}^{\eta_{1}} \cdots \cdots \delta_{n}^{\eta_{n}}$, $\omega=\eta_{1} e^{1}+\cdots+\eta_{n} e^{n}$ and coefficients $c_{\omega} \in \mathbb{C}_{K}[[z]]$.

It is required to find a function $f(x)$ satisfying a polynomial difference equation

$$
P(\delta) f(x)=g(x)
$$


and initial-boundary conditions

$$
\left.\delta^{a^{j} k}[f(x)-\varphi(x)]\right|_{x \in \Gamma_{j} \cap \mathbb{Z}^{n}}=0, \quad 0 \leqslant k<\left\langle m, \alpha^{j}\right\rangle, \quad j=1, \ldots, n,
$$

where $\Gamma_{j}$ is the face of the cone $K$ generated by the vectors $a^{i}, i=1, \ldots, j-1, j+1, \ldots, n$, the vectors $\alpha^{1}, \ldots, \alpha^{n}$ form a reciprocal basis for the vectors $a^{1}, \ldots, a^{n}$, that is, $\left\langle\alpha^{i}, a^{j}\right\rangle=\delta_{i j}$.

Let us state the assertion (see [5], Theorem 1]), which is necessary for the proof of the main result.

Let $c_{\omega}$ be constant, $m \in \Omega$ and let $|m|_{\nu}=d$ be the order of a difference operator. If the coefficients $c_{\omega}$ of the principal symbol $P_{d}(\delta)$ satisfy the condition

$$
\left|c_{m}\right|>\sum_{|\omega|_{\nu}=d, \omega \neq m}\left|c_{\omega}\right|
$$

then (15)-(16) is solvable.

For the case $K \cap \mathbb{Z}^{n}=\mathbb{Z}_{+}^{n}$, Theorem 2 was proved in [8].

For a function $f(x)$ of a discrete argument, we define two types of generating series (functions):

$$
\mathcal{F}(z)=\sum_{x \in K \cap \mathbb{Z}^{n}} \frac{f(x) z^{x}}{\langle x, \alpha\rangle !}
$$

and

$$
F(z)=\sum_{x \in K \cap \mathbb{Z}^{n}} \frac{f(x)}{z^{x}}
$$

The series (18) is called the Borel transform of the series (17). For $K=\mathbb{R}_{+}^{n}$, the standard Borel transform is obtained from the transformation defined above (see $[9,10])$.

We shall look for solutions $\mathcal{F}(z) \in \mathbb{C}_{K}[[z]]$ of the problem (8)-(9) in the form (17).

Theorem 2. The formal Laurent series (17) is the solution of the differential problem (8)-(9) if and only if its Borel transformation (18) is the generating function of the solution $f(x)$ of the difference problem (15)-(16).

Proof. Necessity.

Using the linearity of the differential operator $P(D)$ and the definition of (4) for differentiating $D^{\omega}$, we obtain:

$$
P(D) \mathcal{F}(z)=\sum_{|\omega|_{\nu} \leqslant d} c_{\omega} D^{\omega} \sum_{x \in K \cap \mathbb{Z}^{n}} \frac{f(x) z^{x}}{\langle x, \alpha\rangle !}=\sum_{|\omega|_{\nu} \leqslant d} c_{\omega} \sum_{x-\omega \in K \cap \mathbb{Z}^{n}} \frac{\langle x, \alpha\rangle !}{\langle x-\omega, \alpha\rangle !} \frac{f(x) z^{x-\omega}}{\langle x, \alpha\rangle !} .
$$

After standard transformations, the expression for $P(D) \mathcal{F}$ takes the form

$$
P(D) \mathcal{F}(z)=\sum_{|\omega|_{\nu} \leqslant d} c_{\omega} \sum_{x \in K \cap \mathbb{Z}^{n}} \frac{f(x+\omega) z^{x}}{\langle x, \alpha\rangle !}
$$

Now, changing the order of summation in the last equality and taking into account the equation (8), we obtain:

$$
P(D) \mathcal{F}(z)=\sum_{x \in K \cap \mathbb{Z}^{n}} \sum_{|\omega|_{\nu} \leqslant d} \frac{c_{\omega} f(x+\omega) z^{x}}{\langle x, \alpha\rangle !}=\sum_{x \in K \cap \mathbb{Z}^{n}} \frac{g(x) z^{x}}{\langle x, \alpha\rangle !} .
$$


Finally, equating the coefficients of the same powers, we have

$$
\sum_{|\omega|_{\nu} \leqslant d} c_{\omega} f(x+\omega)=g(x),
$$

that is, $f(x)$ satisfies the difference equation (15).

In general, it is similarly proved that the condition (9) implies the condition (16)

$$
\begin{aligned}
D^{a^{j} k}[\mathcal{F}-\Phi] & =D^{a^{j} k}\left[\sum_{x \in K \cap \mathbb{Z}^{n}} \frac{f(x) z^{x}}{\langle x, \alpha\rangle !}-\sum_{x \in K \cap \mathbb{Z}^{n}} \frac{\varphi(x) z^{x}}{\langle x, \alpha\rangle !}\right]= \\
& =\sum_{x-a^{j} k \in K \cap \mathbb{Z}^{n}} \frac{\langle x, \alpha\rangle !(f(x)-\varphi(x)) z^{x-a^{j} k}}{\left\langle x-a^{j} k, \alpha\right\rangle !\langle x, \alpha\rangle !}=\sum_{x \in K \cap \mathbb{Z}^{n}} \frac{\left(f\left(x+a^{j} k\right)-\varphi\left(x+a^{j} k\right)\right) z^{x}}{\langle x, \alpha\rangle !} .
\end{aligned}
$$

Using the definition of the shift operator $\delta_{j}$, we obtain

$$
D^{a^{j} k}[\mathcal{F}-\Phi]=\sum_{x \in K \cap \mathbb{Z}^{n}} \frac{\delta^{a^{j} k}(f(x)-\varphi(x)) z^{x}}{\langle x, \alpha\rangle !}
$$

then, using the condition (9), we obtain

$$
\left.\sum_{x \in K \cap \mathbb{Z}^{n}} \frac{\delta^{a^{j} k}(f(x)-\varphi(x)) z^{x}}{\langle x, \alpha\rangle !}\right|_{z^{a^{j}}=0}=0 .
$$

According to the definition of (5), we have

$$
\left.\delta^{a^{j} k}[f(x)-\varphi(x)]\right|_{x \in \Gamma_{j} \cap \mathbb{Z}^{n}}=0 .
$$

Sufficiency. If we make the calculations from the proof of necessity in the reverse order, then we get (8) and (9) respectively from (15) and (16).

Proof of Theorem 1.

Since the condition (10) is satisfied, then by the above Theorem 1 from [5] on the solvability of the difference problem (15)-(16) there exists a unique solution $f(x)$. By Theorem 2, the Laurent series $\mathcal{F}=\sum_{x \in K \cap \mathbb{Z}^{n}} \frac{f(x) z^{x}}{\langle x, \alpha\rangle !}$ is the (unique) solution of the problem (8)-(9).

The first author was supported by the grant of the Russian Federation Government for research under the supervision of leading scientist at Siberian Federal University and the State Maintenance Program for the Leading Scientific Schools of the Russian Federation (Grant NSh-9149.2016.1); the second author by RFBR (research project no. 18-31-00232).

\section{References}

[1] N.M.Gunther, On the extension of the Cauchy theorem to any system of partial differential equations, Mat. sb., 32(1925), no. 2, 367-447 (in Russian).

[2] A.G.Khovansky, S.P.Chulkov, The Hilbert and Hilbert-Samuel polynomials and the partial differential equations, Matem. zametki, 7(2005), no. 1, 141-151 (in Russian). 
[3] A.G.Khovansky, S.P.Chulkov, The Hilbert polynomial for systems of linear partial differential equations with analytic coefficients, Izv. RAN. Ser. matem., 70(2006), no. 1, 163-182 (in Russian).

[4] L.Hörmander, Linear differential operators with partial derivatives, Mir, Moscow, 1965 (in Russian).

[5] T.I.Yakovleva, Well-posedness of the Cauchy problem for multidimensional difference equations in rational cones, Siberian Mathematical Journal, 58(2017), no. 2, 363-372.

[6] T.I.Nekrasova, On the Cauchy problem for multidimensional difference equations in rational cone, Journal of Siberian Federal University, Math. and Phys., 8(2015), no. 2, 184-191.

[7] E.K.Leinartas, T.I.Nekrasova, Constant coefficient linear difference equations on the rational cones of the integer lattice, Siberian Mathematical Journal, 57(2016), no. 2, 98-112.

[8] E.K.Leinartas, M.S.Rogozina, Solvability of the Cauchy problem for a polynomial difference operator and monomial bases for the quotients of a polynomial ring, Siberian Mathematical Journal, 56(2015), no. 1, 111-121.

[9] L.Bieberbach, Analytic continuation, Nauka, Moscow, 1967 (in Russian).

[10] L.I.Ronkin, Introduction to the theory of entire functions of several variables, Nauka, Moscow, 1971 (in Russian).

\section{O формальных решениях начально-краевой задачи Хёрмандера в классе рядов Лорана}

\section{Евгений К. Лейнартас}

Татьяна И. Яковлева

Институт математики и фундаментальной информатики

Сибирский федеральный университет

Свободный, 79, Красноярск, 660041

Россия

$\overline{\text { Определяются дифференцирования кольца рядов Лорана с носителями в рациональных конусах и }}$ для полиномиальных дифференииальных операторов с постоянными коэффициентами доказывается теорема существования и единственности решения аналога одной начально-краевой задачи Хёрмандера в классе формальных рядов Лорана.

Ключевые слова: дифференциальный оператор, задача Хёрмандера, разностные уравнения, кратные ряды Лорана. 